\title{
Complications of tracheostomy
}

\author{
Michelle Lee, Henrietta Wilson
}

Barts Thorax Centre, St. Bartholomew's Hospital, West Smithfield, London, UK

Contributions: (I) Conception and design: H Wilson; (II) Administrative support: Both authors; (III) Provision of study materials or patients: H Wilson; (IV) Collection and assembly of data: M Lee; (V) Data analysis and interpretation: Both authors; (VI) Manuscript writing: Both authors; (VII) Final approval of manuscript: Both authors.

Correspondence to: Henrietta Wilson, FRCS, CTh. Barts Thorax Centre, St. Bartholomew's Hospital, West Smithfield, London, UK.

Email: Henrietta.wilson1@nhs.net.

\begin{abstract}
Tracheostomy is a common procedure performed in critically ill patients requiring extended mechanical ventilation for acute respiratory failure and to manage upper airway disorders and obstruction. The recent Coronavirus disease 2019 (COVID-19) pandemic has led to an unprecedented surge in the number of patients who are critically ill and require mechanical ventilation. Tracheostomy is a high-risk procedure in this patient cohort for both the patient and operator due to the need for apnoea at a time of critical illness and the aerosol generating nature of the procedure. Careful consideration of timing, environment, equipment, personnel, and technique are required. In this chapter we will outline the common problems that can occur with tracheostomy during the procedure, in the early stages and late complications. We will highlight both management options for these complications and preventative strategies. The different techniques that can be used including a hybrid surgical and percutaneous approach are described. In addition, the experience of a single surgeon operator during the COVID-19 pandemic, between March and June 2020, who performed a total of 22 tracheostomies on 21 COVID positive patients at St. Bartholomew's hospital: 8 percutaneous dilatational tracheostomies and 14 surgical tracheostomies is presented. We will discuss the outcomes, the specific challenges of performing tracheostomy during a pandemic and how these were overcome.
\end{abstract}

Keywords: Tracheostomy; thoracic surgery; morbidity

Received: 28 May 2021; Accepted: 10 August 2021; Published: 30 October 2021.

doi: $10.21037 /$ shc-21-21

View this article at: https://dx.doi.org/10.21037/shc-21-21

\section{Introduction}

Tracheostomy is a common procedure performed in critically ill patients requiring extended mechanical ventilation for acute respiratory failure and to manage upper airway disorders and obstruction. Use of tracheostomy can facilitate weaning from ventilation and potentially increase the availability of intensive care unit (ICU) beds.

The recent Coronavirus disease 2019 (COVID-19) pandemic has led to an unprecedented surge in the number of patients who are critically ill and require mechanical ventilation. COVID-19 is caused by SARS-CoV-2 virus, which is found in high abundance in the upper aerodigestive tract mucosa (1). It is known to be transmitted via close contact, droplet and aerosols from aerosol generating procedures (AGPs) with tracheostomy being particularly high risk (2).

In this chapter we will outline the common complications of tracheostomy and ways of managing these. In addition, we will present our experience of performing tracheostomy during the COVID-19 pandemic, the specific challenges this posed and how these were overcome.

\section{Indications}

The major indication for tracheostomy is extended mechanical ventilation and respiratory weaning to minimise the complications that occur with prolonged translaryngeal 
endotracheal tube. About $5-10 \%$ of the ICU population require prolonged mechanical ventilation and tracheostomy (3).

Less commonly, tracheostomy may also be required for actual or threatened airway obstruction or laryngeal oedema, which might be an emerging feature of COVID-19 (4). Other indications include adjunct to major head and neck surgery management or airway protection following neurologic disease or traumatic brain injury (Table 1).

\section{Anatomy}

The trachea sits anteriorly in the neck and passes posteriorly through the mediastinum. It is made up of 18-22 C-shaped cartilaginous rings with a membranous posterior wall. On average, the trachea is $2.3 \mathrm{~cm}$ wide and $1.8 \mathrm{~cm}$ from

Table 1 Indications for tracheostomy (5)

Indications for tracheostomy
Prolonged intubation
Facilitation of ventilation support/weaning
More efficient pulmonary hygiene (i.e., managing secretions)
Upper airway obstruction
Stridor, air hunger, retractions
Obstructive sleep apnoea with documented arterial
desaturation
Bilateral vocal cord paralysis
Inability to intubate
Adjunct to major head or neck surgery/trauma management
Airway protection (neurological disease, traumatic brain injury)

the anterior cartilage to the posterior membrane (6). The Trachea is protected by bony structures (sternum and manubrium) and by strap muscles (sternohyoid, sternothyroid and sternocleidomastoid). The blood supply is provided by branches of the bronchial, inferior thyroid, innominate and subclavian arteries (Figure 1).

When positioning the tracheostomy, percutaneous tubes are typically placed between the first and second or the second and third tracheal cartilaginous rings. Surgical tracheostomy (ST) tubes are typically placed between second to fourth tracheal rings.

\section{Technical approach}

Tracheostomy is the process of creating an opening in the anterior wall of trachea. The two main approaches are open ST and percutaneous dilatational tracheostomy (PDT).

\section{PDT}

PDT is mostly performed in ICU, where the patient is already intubated and sedated, but the challenging cases may be performed in an operating theatre. PDT uses a Seldinger technique, in which the trachea is accessed with a needle, then a guidewire is inserted. Following dilatation of the trachea, tracheostomy tube is introduced over the guidewire (Figure 2).

Patient selection is a significant factor in considering which technique to use. Unstable patients or those with infections of the anterior neck are absolute contraindications for tracheostomy and some patients may require an open approach due to anatomical or other concerns. Tables 2,3 describe critically important pre-operative criteria and

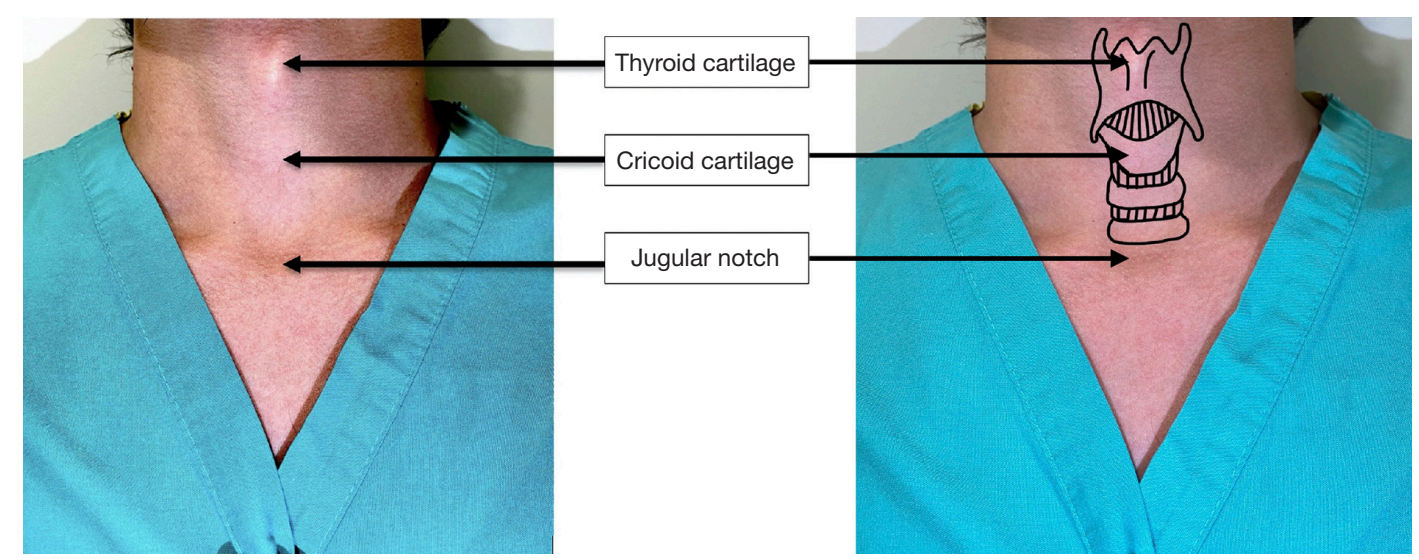

Figure 1 Surface anatomy of anterior neck. 


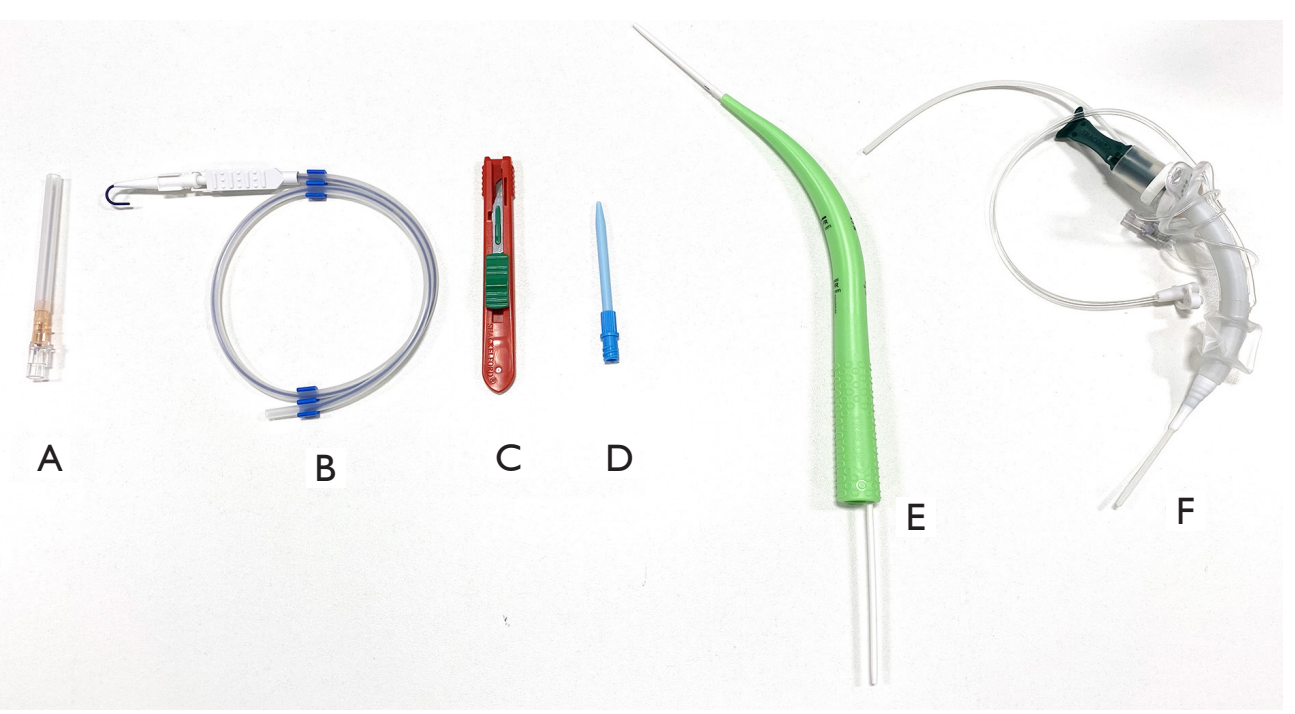

Figure 2 PDT Set. (A) Needle; (B) guidewire; (C) scalpel; (D) small dilator; (E) large dilator; (F) tracheostomy tube. PDT, percutaneous dilatational tracheostomy.

Table 2 Critically important pre-operative criteria for PDT $(7,8)$

Ability to hyperextend the neck

Presence of at least $1 \mathrm{~cm}$ distance between the inferior cricoid cartilage and the suprasternal notch

Patient tolerance of transient hypercarbia and hypoxaemia

PDT, percutaneous dilatational tracheostomy.

contraindications to be considered for PDT.

\section{$S T$}

ST is considered for patients with contraindications to PDT and involves dissection of the pretracheal tissues and incision through the anterior tracheal wall, followed by placement of tracheostomy cannula under direct vision. Several different incisions are commonly used for ST.

\section{Horizontal incision}

This involves a horizontal incision midway between the cricoid cartilage and the sternal notch and is the least traumatic approach to the cartilage but may cause difficulties in inserted the tracheostomy tube.

\section{Tracheal window}

Instead of a simple horizontal incision, the midportion of the third or fourth tracheal ring can be incised to create a window. The theoretical rationale for the tracheal window is to minimise trauma to the remaining cartilage from passage of the tracheostomy tube but may increase the risk of tracheal stenosis (9).

\section{Bjork flap}

A Bjork flap is an inferiorly based U-shaped flap, which is created in the anterior trachea by two vertical incisions from the lateral extents of the tracheotomy. The theoretical justification for the use is to keep the tracheal incision close to the skin edge and facilitate tube replacement before maturation (10).

\section{Hybrid surgical tracheostomy (Hybrid ST)}

A Hybrid ST is a combined surgical approach as far as the anterior tracheal wall followed by Seldinger technique through the trachea. A guidewire is inserted between the cartilaginous rings rather than dividing the cartilage. This approach can therefore be use for the patients who are not suitable for PDT but maintains the integrity of the tracheal cartilaginous rings.

\section{Complications of tracheostomy}

Complications of tracheostomy can be considered occurring in three-time frames: immediate, early (0-7 days of procedure) or late (beyond day 7) (Table 4). 
Table 3 Contraindications of PDT $(7,8)$

\begin{tabular}{|c|}
\hline Absolute \\
\hline Infants \\
\hline Infection at insertion site \\
\hline Unstable cervical spine injury \\
\hline Uncontrollable coagulopathy \\
\hline Operator inexperience \\
\hline Relative \\
\hline Emergency airway access \\
\hline Difficult anatomy \\
\hline Morbid obesity with short neck \\
\hline Limited neck movement \\
\hline Cervical spine injury \\
\hline Aberrant blood vessels \\
\hline Thyroid or tracheal pathology \\
\hline Coagulopathy \\
\hline $\begin{array}{l}\text { Prothrombin time or activated partial thromboplastin time } \\
>1.5 \text { times reference range }\end{array}$ \\
\hline Thromcobytopaenia, platelet count $<50,000$ cells $/ \mu \mathrm{L}$ \\
\hline Significant gas exchange problems \\
\hline Positive end-expiratory pressure (PEEP) $>10 \mathrm{~cm} \mathrm{H}_{2} \mathrm{O}$ \\
\hline $\mathrm{FiO}_{2}>0.6$ \\
\hline $\begin{array}{l}\text { Need for proximal or distal extension tracheostomy tube } \\
\text { placement }\end{array}$ \\
\hline
\end{tabular}

PDT, percutaneous dilatational tracheostomy.

\section{Immediate complications of tracheostomy}

\section{Bleeding}

Bleeding is the most common early complication of tracheostomy and the incidence of major or minor bleeding following tracheostomy is approximately 5.7\% (13). Although major bleeding during tracheostomy is rare, minor bleeding can be life threatening if it results in airway obstruction. Bleeding may occur perioperatively, in the early post procedural course or later secondary to formation of a fistula. Late haemorrhage is generally due to the tracheostomy tube eroding through the wall of the trachea or a major vessel. Factors contributing to erosion are excessively long or angulated tubes and prolonged cuff pressure (14).

Bleeding is usually related to the vasculature in the field and in particular the thyroid vessels or the gland itself; rarely, severe bleeding can occur due to damage to major vessels such as the innominate artery. Evaluation of the neck vasculature with ultrasound can be used to identify any significant vessels prior to procedure. Presence of significant vessels in the field is not a contraindication to percutaneous tracheostomy, but in this situation, it may be preferable to perform the procedure in the operating theatre so that bleeding can be managed more proficiently with the option to change to a surgical approach if needed. During the procedure, meticulous haemostasis or use of the Seldinger technique through the anterior tracheal wall can reduce bleeding from the trachea.

Superficial bleeding can be managed by local measures such as applying local pressure or light packing with adrenaline or tranexamic acid-soaked gauze. However, ongoing bleeding after tube placement should always

Table 4 Complications of tracheostomy $(11,12)$

\begin{tabular}{lll}
\hline Immediate & Early & Late \\
\hline$*$ Haemorrhage & $*$ Haemorrhage & $*$ Tracheal stenosis \\
$*$ Damage to nearby structures & $*$ Tube displacement & $*$ Tracheomalacia \\
$*$ Air embolism & $*$ Pneumothorax & $*$ Pneumomediastinum \\
$*$ Aspiration & $*$ Subcutaneous emphysema tissue \\
$*$ Loss of airway & $*$ Stomal infection & $*$ Aspiration \\
$*$ Hypoxaemia, hypercarbia & $*$ Stomal ulceration & $*$ Tracheovascular fistula \\
$*$ Death & $*$ Accidental decannulation & $*$ Accidental decannulation \\
& $*$ Dysphagia & $*$ Dysphagia \\
\hline
\end{tabular}


be investigated thoroughly and most require surgical exploration. Preventative measures include performing tracheostomy at or above third tracheal ring, using a tube with adequate length and use of ultrasound to identify major vessels. Following tube placement, if pulsation is present, changing the tube position may be required or a shorter tube can be placed (14).

\section{Loss of airway}

Loss of airway with hypoxia is another life-threatening complication of tracheostomy and requires immediate intervention. The incidence of tracheostomy tube obstruction following PDT occurs in $0-3.5 \%$ of cases. It may present with acute deterioration of respiratory status and could result in death. It can be due to mucus plugging or clot within the airway or passage of the tracheostomy tube into a false lumen (15). Mucous plugs can also be aspirated and lead to atelectasis or lung abscess.

Obstruction due to mucous or clot can be immediately relieved by suctioning of tracheostomy tube or exchanging the inner tube. Furthermore, choosing appropriate tube size and horizontal length based on the patient's anatomy can reduce the risk of obstruction from the posterior tracheal wall (16). To avoid passage into a false lumen, a guidewire can be used during the change, or this can be performed under bronchoscopic guidance.

Loss of airway during ST can occur when inserted the tracheostomy tube through the tracheal window. This can be managed by ensuring the ET tube is not removed until the tracheostomy airway is secure. We would also advise having a size 6 endotracheal tube ready in theatre which can be passed easily through the tracheal window in an emergency. A suture to stabilise the anterior tracheal wall and provide traction can also be helpful. Alternatively, Seldinger hybrid approach can be used to reduce risks of this problem by inserting a tube over a guidewire and dilating the trachea to the correct size, rather than approximation.

\section{Damage to trachea}

Injury to the posterior tracheal wall can occur during PDT and it is caused by poor control of the guidewire and guiding catheter.

Most posterior tracheal wall tears are small and do not require further intervention. Larger tears, however, may be life-threatening and may require emergency surgical intervention. Larger tears may present with airway bleeding or air leak into the mediastinum and surgical emphysema.
Surgical repair of large tears may require the use of cardiopulmonary bypass. This may not be feasible in critically ill patients and therefore a self-expanding metallic stent may be considered (17).

\section{Early complications of tracheostomy}

\section{Stomal infection}

Local infection occurs in approximately $5 \%$ of tracheostomies and most commonly occur at the stoma, the tip of the tube and the area of the cuff. This is more common following a surgical approach (14). Predisposing factors include a large stoma, chemicals such as a strong antiseptic used for cleaning or ischaemia secondary to cuff pressure (18).

The risk can be reduced by avoiding a large neck incision, regular dressing changes and assessment on dedicated tracheostomy ward rounds. Prophylactic antibiotics are not commonly recommended prior to procedure. For minor stomal infection, appropriate wound care is generally sufficient, but rarely severe cases such as necrotising tracheal infection will require oral tracheal intubation followed by debridement of involved tissue (16).

\section{Tube displacement}

Tracheostomy tube displacement within 7 days of procedure is a medical emergency, resulting in loss of airway and need for emergency intubation. However, the absence of a mature tracheostomy tract may make replacement of the tube difficult and lead to the creation of a false tract. Incidence of tube displacement after PDT is approximately $1 \%$ and is likely to occur if tracheostomy tube is placed too low or not in the midline. Other possible causes include loose tracheostomy ties, self-extubation or accidental displacement while turning the patient. The risk is higher in obese patients, as skin to tracheal distance may be more than $2-4 \mathrm{~cm}$. This can be minimised by using an adjustable flange tracheostomy tube or restricting first tracheostomy tube change until after 5-7 days (16).

If the tube is accidentally dislodged oral endotracheal intubation should be carried out to secure the airway. The tracheostomy can then be reinserted using a guiding catheter or bronchoscopic guidance to reduce the risk of paratracheal insertion (16).

\section{Subcutaneous emphysema}

Incidence of subcutaneous emphysema range from $0-5 \%$ and it is caused by formation of tissue tract anterior to 


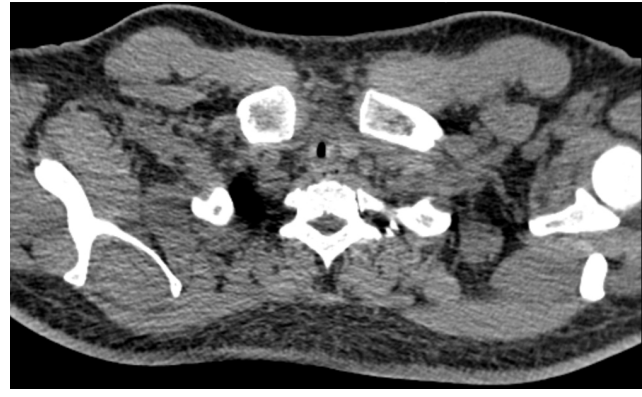

Figure 3 CT scan showing post tracheostomy tracheal stenosis. CT, computer tomography.

trachea, secondary to positive pressure ventilation or forced coughing against a tightly packed or occluded neck dressing. Expired air escaping from the trachea may dissect through the subcutaneous tissue into the neck or through the pretracheal fascia into the mediastinum. In addition, air may extend into the pleural space leading to development of pneumothorax (16).

This is typically treated conservatively and resolved spontaneously by releasing the skin sutures.

\section{Pneumothorax}

The Incidence of pneumothorax is higher in children following tracheostomy; this may be because pleural domes in children lie higher in the neck and therefore are more prone to the injury. Another cause is pneumomediastinum, which may lead to ruptured pleura and pneumothorax. This can again occur more commonly in children due to loose tissues in the neck and therefore it is easier for air to be sucked through the tracheostomy wound. To reduce risk, minimal dissection of the pretracheal fascia is recommended (14).

Significant pneumothorax following tracheostomy should be managed with insertion of an intercostal drain.

\section{Late complications of tracheostomy}

\section{Tracheal stenosis}

Tracheal stenosis is the most common serious late complication of tracheostomy and is defined as abnormal narrowing of the tracheal lumen due to either fibrosis or granulation tissue (Figure 3). The pathogenesis of tracheal stenosis involves inflammation and the formation of granulation tissue. Predisposing factors such as trauma from tracheostomy, mucosal ischaemia from excessive cuff pressures or persistent mucosal irritation lead to development of ulceration and inflammation of cartilage and ultimately lead to formation of granulation tissue. Granulation tissue then matures into a fibrous tissue layer covered with epithelium leading to tracheal stenosis (16). Other risk factors include male gender, advanced age, stomal infection or tight, oversized cannula (19).

All patients following tracheostomy may have a certain degree of tracheal stenosis, but it only usually becomes symptomatic after involvement $>50 \%$ of lumen. The incidence of clinically significant tracheal stenosis following tracheostomy is $3-12 \%$. Tracheal stenosis may be detected while the patient is still mechanically ventilated leading to difficulty in weaning from the ventilator. Onset of symptoms vary between days to months after decannulation, but typically dyspnoea, stridor or respiratory failure are present within two months following decannulation (16).

Correct classification of stenosis is essential to determine appropriate management and flexible bronchoscopy is the gold standard for diagnosis. It allows direct visual evaluation of the stenosis and classification can be determined based on type, size, location, and degree of stenosis. Types include simple, complex stenosis or cartilage damage. Size refers to the vertical length in centimetres. Location is defined as tracheal, supraglottic, glottic or subglottic. Degree of stenosis is based on percentage of obstructed lumen; grade $1<50 \%$, grade 250 $70 \%$, grade $371-99 \%$ and grade $4100 \%$ of obstruction (20). Other non-invasive testing includes computer tomography (CT) scan, which can predict the size or location of stenosis. Pulmonary function testing can also identify fixed airway obstructions, but this may not detect small changes in tracheal lumen in a diameter above $8 \mathrm{~mm}$ (21).

Management of tracheal stenosis may be endobronchial with laser treatment or dilatation. In some cases, surgical resection of the stenotic portion of trachea may be required. An endoscopic approach using laser is the most common initial treatment option (Figure 4).

If endobronchial management is unsuccessful, the patient will require surgical resection. If subglottic tracheal lesions involve the larynx, laryngotracheal reconstruction will be required. To reduce the incidence of tracheal stenosis the operator should avoid opening the trachea above the second tracheal ring or excessive damage to the anterior wall. During ventilation, the cuff pressure should not exceed $22 \mathrm{mmHg}$ and should be checked by trained health professional regularly (14).

\section{Tracheomalacia}

Tracheomalacia is a rare complication, which is characterised 


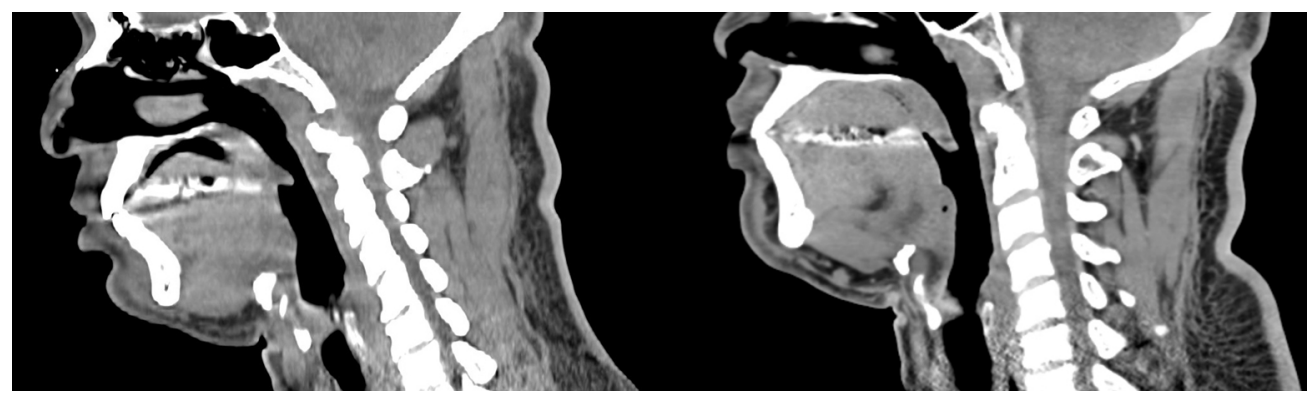

Figure 4 Sagittal CT of the neck: pre \& post-laser treatment of tracheal stenosis. CT, computer tomography.

as the loss of cartilage structure in the tracheal wall leading to dynamic expiratory collapse and airway obstruction. During forced expiration, there is a reduction of $>50 \%$ in crosssection area of trachea. There are several predisposing factors including ischaemia from elevated tracheostomy tube cuff pressures and recurrent episodes of gastroesophageal reflux during mechanical ventilation leading to inflammation of the tracheal rings (chondritis). Prolonged chondritis may then lead to weakening of the tracheal rings resulting in airway deformity, increased airway compliance and ultimately hyperdynamic airway collapse during expiration (16).

Clinical symptoms of tracheomalacia include exertional dyspnoea, cough, recurrent pulmonary infections, and haemoptysis. These may be present months to years after decannulation. In addition, unexplained difficulty in weaning from the ventilator needing for re-intubation following decannulation is a significant clinical sign of tracheomalacia. Dynamic flexible bronchoscopy remains the gold standard for diagnosis, but CT chest and pulmonary function testing can also be useful (16).

For management of severe tracheomalacia posterior membrane tracheoplasty or surgical intervention, such as focal tracheal resection and reconstruction can be considered (16).

\section{Tracheovascular fistula}

Tracheo-innominate artery fistula (TIF) is an uncommon $(<1 \%)$, but fatal complication which usually occurs 3-4 weeks after tracheostomy. TIFs occur due to erosion into the innominate artery by the tracheostomy tube due to high cuff pressure or contact between the distal end of the tube and the innominate artery. Other risk factors include low tracheostomy insertion or repetitive head movements (16).

Although digital pressure on the anterior wall of the stoma can be a temporary measure, management of possible fistula should take place in the operating theatre. A rigid bronchoscope can be used to assess the stoma and anterior wall, then tracheostomy tube should be removed. In case of severe bleeding, the patient will require emergency median sternotomy with ligation of innominate artery and tracheal wall repair (22). The risk of vascular fistula can be reduced by correct placement of the tracheostomy tube in between the first and the second or second and the third tracheal rings. The risk is higher if the tube is placed in a more inferior position.

\section{Tracheoesophageal fistula}

Transoesophageal fistula (TEF) is a very rare, but lifethreatening complication. It is most commonly seen in the patients with nasogastric tubes, and it occurs secondary to increased tracheostomy cuff pressures and subsequent ischaemia of the tracheal mucosa. Prolonged ischaemia can then lead to tracheal wall necrosis, ulceration and perforation with erosion into the oesophagus. Other risk factors for TEF include injury to the posterior tracheal wall during stoma creation, during intubation or erosion of the tracheostomy cuff through the posterior tracheal wall due to excessive cuff pressure (19).

Immediate management involves passing a long-cuffed tube beyond the level of the fistula. Timing of surgical correction can be difficult as mechanically ventilated patients are not candidates for surgical correction. In those patients who are no longer ventilated and are in a stable clinical condition, surgical correction may be performed to separate the oesophagus from the trachea and close the defects. A muscle flap is usually required to protect the repaired area. For patients who are not suitable for surgical intervention a combination of tracheal and oesophageal stenting may be used for palliative measures.

\section{Performing tracheostomy in the COVID era}

The COVID-19 pandemic has created an environment in 
Table 5 Core team members for tracheostomy $(23)$

\begin{tabular}{ll}
\hline ST & PDT \\
\hline$*$ Consultant surgeon & $*$ Consultant surgeon \\
$*$ Consultant anaesthetist & $*$ Consultant anaesthetist \\
$*$ Skilled surgical assistant & $*$ Assistant for airway manager \\
$*$ Scrub nurse & $*$ Appropriately skilled individual in fibreoptic bronchoscopy \\
$*$ Second theatre nurse as runner (positioned outside the room) & $*$ Runner outside the room \\
\hline ODP
\end{tabular}

ST, surgical tracheostomy; PDT, percutaneous dilatational tracheostomy; ODP, operating department practitioner.

which an increased number of critically ill patients require tracheostomy for respiratory weaning, while performing the procedure itself poses additional risks to healthcare workers. Tracheostomy is a high-risk procedure in this patient cohort for both the patient and operator due to the need for apnoea at a time of critical illness and the aerosol generating nature. Careful consideration of timing, environment, equipment, personnel, and technique are required.

\section{Planning tracheostomy for COVID positive patients}

\section{Planning the optimal setting for tracheostomy}

Tracheostomy should be planned as a semi-elective case with an experienced theatre team and anaesthetist. During the COVID-19 pandemic we tried to limit the number of staff members who carried out these procedures to increase experience and communication and reduce the potential risks and complications.

Percutaneous, surgical or hybrid approach can all be used either at the bedside or in an operating theatre. In our experience, where the anatomy was favourable for a percutaneous approach, these were carried out at the bedside in ICU by experienced anaesthetists and intensive care specialists. Where the anatomy was not favourable or in patients with higher oxygen requirements the procedure was performed in a temporary operating theatre created within the IUC and with facilities to perform a surgical approach if needed. This allowed for a superior environment to the bedside without transferring the patient to the main operating theatre which may carry exposure risks to multiple patients and staff.

\section{COVID airway team}

Tracheostomy is a high-risk AGP and it may represent a significant risk to healthcare professionals. It is therefore important that only core members of staff are present for the procedure (Table 5).

\section{Personal protective equipment (PPE)}

When performing AGPs, such as tracheostomy, eye protection, filtering face piece 3 (FFP3) respirator, long sleeved fluid repellent gown and gloves should be worn (Figure 5).

During tracheostomy in COVID-19 positive patients, those staff members within the operating room required a surgical helmet Stryker hood to provide additional protection against liquid and viral contamination (Figure 6).

\section{Performing tracheostomy for COVID positive patients}

Specific techniques were employed to reduce the risk to members of the surgical team involved in tracheostomy in COVID-19 positive patients. The overall aim is to reduce the aerosolization risk without life-threatening periods of apnoea. The technique varies depending on whether a surgical or percutaneous approach is used and are outlined in the tables below (Tables 6,7).

\section{Complications in patients with COVID}

We present the experience of a single surgeon operator during the COVID-19 pandemic, between March and June 2020, who performed a total of 22 tracheostomies on 21 COVID positive patients at St. Bartholomew's hospital: 8 percutaneous dilatational tracheostomies (PDT) and 14 surgical tracheostomies (ST).

\section{PDT (Table 8)}

In total eight patients who underwent PDT, no complications 


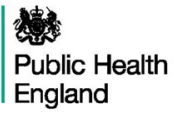

COVID-19 Safe ways of working A visual guide to safe PPE

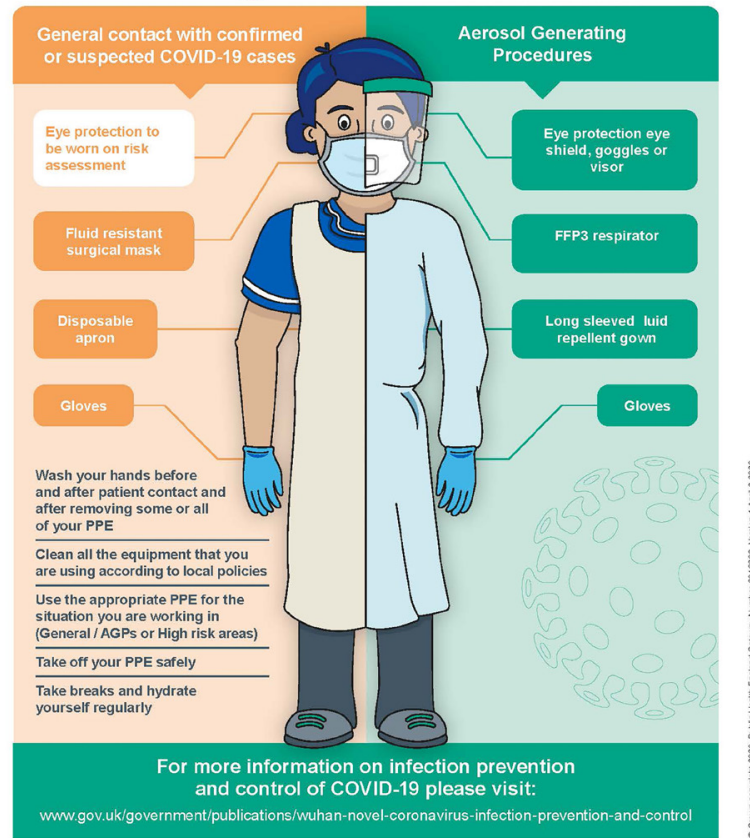

Figure 5 COVID-19: a visual guide to safe PPE by Public Health England (24). PPE, personal protective equipment.

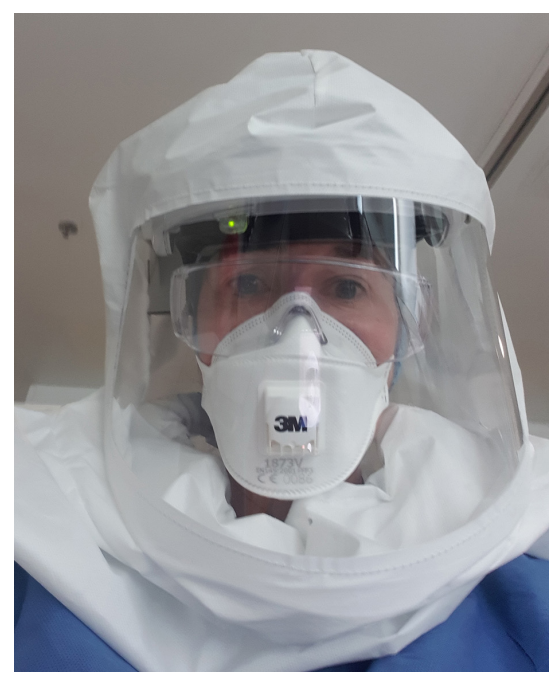

Figure 6 Miss Henrietta Wilson, thoracic consultant wearing Stryker hood for tracheostomy. This image is published with the participant's consent. 
Table 7 ST technique (23)

* Dissection to trachea

* Prior to making tracheal window

- Advance ETT to position below window

- Pre-oxygenate

- Give additional dose muscle relaxant

- Stop ventilation in case of cuff rupture. Ventilation can be recommenced following dissection through the trachea if cuff intact

* Once window prepared and rescue suture in place

- Suction oropharynx

- Stop ventilation

- Deflate cuff and withdraw ETT under the direction of the surgeon

- Insert tracheostomy

- If unable to insert tracheostomy, re-advance ETT and reinflate cuff prior to recommencing IPPV

- Once tracheostomy in situ, inflate cuff, connect in-line suction (rather than standard catheter mount), HME and ventilator tubing

- Commence ventilation

- Confirm placement by observing capnography and bilateral chest movement

* Do not auscultate unless specific concern

* Suction trachea using closed in-line suction

ST, surgical tracheostomy; ETT, endotracheal tube; IPPV, intermittent positive pressure ventilation; HME, heat moisture exchanger.

related to the procedure were reported to date.

\section{ST (Table 9)}

In total 14 COVID positive patients who underwent ST, majority $64 \%(n=9)$ had uncomplicated procedures. Two patients had minor immediate complications: one patient had cuff puncture during the procedure, but this was clinically insignificant. Another patient had minor bleeding which was managed with sutures at the bedside in ICU.

During the early period (0-7 days of procedure), one patient developed cuff leak on day 6 and which resolved following upsizing of the tracheostomy tube using a
Table 8 PDT at St. Bartholomew's Hospital $(n=8)$ during COVID pandemic

\begin{tabular}{lccc}
\hline \multirow{2}{*}{ Uncomplicated } & \multicolumn{3}{c}{ Complications } \\
\cline { 2 - 4 } & Immediate & Early & Late \\
\hline 8 & 0 & 0 & 0 \\
\hline \multicolumn{2}{l}{ PDT, percutaneous dilatational tracheostomy. }
\end{tabular}

Table 9 ST at St. Bartholomew's Hospital $(n=14)$ during covid pandemic

\begin{tabular}{lccc}
\hline \multirow{2}{*}{ Uncomplicated } & \multicolumn{3}{c}{ Complications } \\
\cline { 2 - 4 } & Immediate & Early & Late \\
\hline 9 & 2 & 1 & 2 \\
\hline ST, surgical tracheostomy. & &
\end{tabular}

Seldinger technique.

During the late period (beyond day 7 post-procedure), two patients were found to have tracheal stenosis. Both were managed with successful microlaryngoscopy, and laser treatment then discharged home.

There were no cases of COVID-19 reported within the healthcare team carrying out these procedures.

\section{Conclusions}

Tracheostomy is a commonly performed procedure but can lead to life-threatening complications. Patient characteristics and operator experience should guide the technical approach, but we would advocate the use of the hybrid technique where a full percutaneous approach is not possible to maintain the integrity of the anterior tracheal wall. Increases in the number of critically ill patients during the COVID-19 pandemic saw an increase in the number of tracheostomies required to facilitate recovery. The COVID-19 pandemic created unique challenges for those performing this procedure but with careful planning and the correct protective equipment, tracheostomy can be carried out safely with minimal risk to patients and hospital staff.

\section{Acknowledgments}

Funding: None.

\section{Footnote}

Provenance and Peer Review: This article was commissioned 
by the Guest Editor (David Waller) for the series "Complications of Thoracic Surgery-aetiology, management and prevention" published in Shanghai Chest. The article has undergone external peer review.

Conflicts of Interest: Both authors have completed the ICMJE uniform disclosure form (available at: http://dx.doi. org/10.21037/shc-21-21). The series "Complications of Thoracic Surgery-aetiology, management and prevention" was commissioned by the editorial office without any funding or sponsorship. Both authors have no other conflicts of interest to declare.

Ethical Statement: The authors are accountable for all aspects of the work in ensuring that questions related to the accuracy or integrity of any part of the work are appropriately investigated and resolved.

Open Access Statement: This is an Open Access article distributed in accordance with the Creative Commons Attribution-NonCommercial-NoDerivs 4.0 International License (CC BY-NC-ND 4.0), which permits the noncommercial replication and distribution of the article with the strict proviso that no changes or edits are made and the original work is properly cited (including links to both the formal publication through the relevant DOI and the license). See: https://creativecommons.org/licenses/by-ncnd/4.0/.

\section{References}

1. Zou L, Ruan F, Huang M, et al. SARS-CoV-2 Viral Load in Upper Respiratory Specimens of Infected Patients. N Engl J Med 2020;382:1177-9.

2. Infection Control: Severe acute respiratory syndrome coronavirus 2 (SARS-CoV-2) [Internet]. Centers for Disease Control and Prevention. Centers for Disease Control and Prevention; [cited 2021 Sep4]. Available online: https://www.cdc.gov/coronavirus/2019-ncov/hcp/ infection-control-recommendations.html

3. Cohen IL, Booth FV. Cost containment and mechanical ventilation in the United States. New Horiz 1994;2:283-90.

4. McGrath BA, Wallace S, Goswamy J. Laryngeal oedema associated with COVID-19 complicating airway management. Anaesthesia 2020;75:972.

5. De Leyn P, Bedert L, Delcroix M, et al. Tracheotomy: clinical review and guidelines. Eur J Cardiothorac Surg
2007;32:412-21.

6. Rood S. Anatomy for tracheotomy. In: Myers E, Stool SE, Johnson JT, editors. Tracheotomy. New York: Churchill Livingstone, 1985:89-97.

7. Huang CS, Chen PT, Cheng SH, et al. Relative contraindications for percutaneous tracheostomy: from the surgeons' perspective. Surg Today 2014;44:107-14.

8. Cheung NH, Napolitano LM. Tracheostomy: epidemiology, indications, timing, technique, and outcomes. Respir Care 2014;59:895-915.

9. Scurry Jr WC, McGinn JD. Operative tracheotomy. Oper Tech Otolayngol Head Neck Surg 2007;18:85-9.

10. Souba, WW, American College of Surgeons. ACS surgery: principles \& practice. New York: WebMD, 2008.

11. Durbin CG Jr. Early complications of tracheostomy. Respir Care 2005;50:511-5.

12. Epstein SK. Late complications of tracheostomy. Respir Care 2005;50:542-9.

13. Delaney A, Bagshaw SM, Nalos M. Percutaneous dilatational tracheostomy versus surgical tracheostomy in critically ill patients: a systematic review and meta-analysis. Crit Care 2006;10:R55.

14. Mehta C, Mehta Y. Percutaneous tracheostomy. Ann Card Anaesth 2017;20:S19-25.

15. Trottier SJ, Ritter S, Lakshmanan R, et al. Percutaneous tracheostomy tube obstruction: warning. Chest 2002;122:1377-81.

16. Fernandez-Bussy S, Mahajan B, Folch E, et al. Tracheostomy Tube Placement: Early and Late Complications. J Bronchology Interv Pulmonol 2015;22:357-64.

17. Madden BP, Sheth A, Ho TB, et al. Novel approach to management of a posterior tracheal tear complicating percutaneous tracheostomy. Br J Anaesth 2004;92:437-9.

18. Pelaez-Fernandez J, Asensio Martin MJ, Jimenez Lendinez M. Percutaneous versus standard tracheostomy. Surgery 1997;121:112-3.

19. Cipriano A, Mao ML, Hon HH, et al. An overview of complications associated with open and percutaneous tracheostomy procedures. Int J Crit Illn Inj Sci 2015;5:179-88.

20. Boiselle PM, Lee KS, Ernst A. Multidetector CT of the central airways. J Thorac Imaging 2005;20:186-95.

21. Miller RD, Hyatt RE. Evaluation of obstructing lesions of the trachea and larynx by flow-volume loops. Am Rev Respir Dis 1973;108:475-81.

22. Sue RD, Susanto I. Long-term complications of artificial airways. Clin Chest Med 2003;24:457-71. 
23. St. Bartholomew's Hospital Tracheostomy Policy in COVID-19. London: St Bartholomew's Hospital; 2021.

24. England PH. COVID-19: infection prevention and control

doi: $10.21037 /$ shc-21-21

Cite this article as: Lee $\mathrm{M}$, Wilson $\mathrm{H}$. Complications of tracheostomy. Shanghai Chest 2021;5:42.
(IPC). GOV.UK. GOV.UK; 2020. Available online: https://www.gov.uk/government/publications/wuhannovel-coronavirus-infection-prevention-and-control 